\title{
An Improved Dynamical Formulation for Constant Temperature and Pressure Dynamics, with Application to Particle Fluid Models
}

\author{
Benedict J. Leimkuhler \\ Department of Mathematics and Computer Science, University of Leicester, \\ Leicester,LE1 7RH, UK \\ bl12@mcs.le.ac.uk
}

\begin{abstract}
A new fully dynamical scheme for constant temperature and pressure simulation of particle systems, based on a modification of the Nosé thermostat. A mechanical formulation for simultaneous control of temperature and pressure is also presented. This approach simplifies the construction of symplectic methods while providing a more intuitive perspective on the nature of controlled variable molecular dynamics. Moreover, the described method is Gallilean-invariant, hence angular momentum preserving, and the per-timestep simulation costs are similar to the per-timestep costs of microcanonical $(N, V, E)$ simulation. The scheme is suited to molecular simulation and to large scale particle models of fluids such as smooth particle hydrodynamics and dissipative particle models.
\end{abstract}

